\title{
On the effective temperatures and surface gravities of superficially normal main sequence band $B$ and $A$ stars
}

\author{
Saul J. Adelman ${ }^{1,2}$, O. I. Pintado ${ }^{3,4}$, F. Nieva ${ }^{3}$, K. E. Rayle ${ }^{1}$, and S. E. Sanders, Jr. ${ }^{1}$ \\ 1 Department of Physics, The Citadel, 171 Moultrie Street, Charleston, SC 29409, USA \\ 2 Guest Investigator, Dominion Astrophysical Observatory, Herzberg Institute of Astrophysics, \\ National Research Council of Canada, 5071 W. Saanich Road, Victoria V9E 2E7 Canada \\ 3 Departamento de Fisica, Facultad de Ciencias Exactas y Tecnologia, Universidad Nacional de Tucumán, \\ Av. Independencia 1800, 4000 San Miguel de Tucumán, Argentina \\ ${ }^{4}$ Member of the Carrera del Investigador Científico, CONICET, Consejo Nacional de Investigaciones Científicas y Técnicas \\ de la República Argentina
}

Received 26 February 2002 / Accepted 12 June 2002

\begin{abstract}
Effective temperatures and surface gravities for 48 main sequence band B and A stars were found by matching optical region spectrophotometry and $\mathrm{H} \gamma$ profiles with the predictions of ATLAS9 solar composition model atmospheres. When these values were compared with those found using Strömgren $u v b y \beta$ photometry based on ATLAS6 model atmospheres, we found a difference (photometry-spectrophotometry) of $25 \pm 118 \mathrm{~K}$ for 29 stars with $8000 \mathrm{~K} \leq T_{\text {eff }} \leq 10050 \mathrm{~K}$ compared to $76 \pm 105 \mathrm{~K}$ for 14 stars with $10050 \mathrm{~K} \leq T_{\text {eff }} \leq 17000 \mathrm{~K}$. The surface gravity scales are in agreement. These stars are sufficiently hot that their effective temperatures and surface gravity determinations are unaffected by discrepancies due to the choice of Mixing-Length or Canuto-Mazzitelli convection theories.
\end{abstract}

Key words. stars: fundamental parameters - stars: early-type

\section{Introduction}

Astronomers can determine the effective temperatures and surface gravities of single main sequence band B and A stars by matching their optical region flux distributions as measured spectrophotometrically and their observed $\mathrm{H} \gamma$ profiles with the predictions of the best model atmospheres. When these observations are not available, investigators have used photometric indices calibrated by this method, e.g. for Strömgren $u v b y \beta$ photometry the work of Moon \& Dworetsky (1985), which has been updated by Napiwotzki et al. (1993). The errors are of order $\pm 200 \mathrm{~K}$ and \pm 0.2 dex in the middle B to early F star regime (Lemke 1989) (see Ribas et al. 1997 concerning which values are better). Our current ability to calculate physically realistic model atmospheres is the greatest in this region of the HR Diagram. These calibrations are based mainly on model atmospheres used prior to 1992 . In the optical region, there can be small differences in the predicted fluxes between ATLAS9 (Kurucz 1993) and the older ATLAS6 (Kurucz 1979) models due in part to an improved treatment of line blanketing and to the use of opacity distribution functions with different microturbulences. This paper to a large extent investigates the effects of these differences.

Send offprint requests to: Saul J. Adelman,

e-mail: adelmans@citadel.edu
In determining stellar effective temperatures and surface gravities, two basic concerns are the agreement of the predictions of the models with the observations as this is what many abundance workers use to select appropriate models and whether these results show systematic errors when one uses flux observations covering a much wider range of wavelengths. In this paper we are concerned mainly with the former. One can obtain infrared and perhaps ultraviolet fluxes and use the results of this study as a basis for determining effective temperatures via the infrared flux method. But for single and binary stars with sufficiently faint companions if there are no systematic errors in the model fluxes, our approach should yield as good results without the complications of additional sources of error.

The ATLAS9 code produces fully line blanketed LTE plane parallel model atmospheres. From converged model atmospheres, one can calculate the fluxes using ATLAS9 and the $\mathrm{H} \gamma$ region using the synthetic spectrum code SYNTHE (Kurucz \& Avrett 1981). For comparison with observations the synthesized spectra were convolved with the measured stellar rotational velocity and the instrumental profile of the short camera of the coudé spectrograph of the Dominion Astrophysical Observatory (DAO) 1.22-m telescope. Trends in recent elemental abundance studies indicate for main sequence band stars with temperatures greater than $10250 \mathrm{~K}$ their 
microturbulence is $0 \mathrm{~km} \mathrm{~s}^{-1}$, for those with temperatures be-

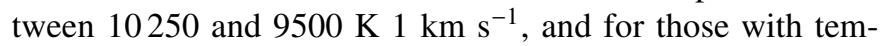

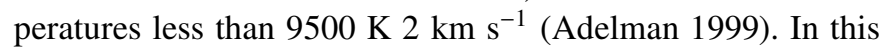
investigation, grids of ATLAS 9 models with effective temperature and surface gravity spacings of $500 \mathrm{~K}$ and $0.25 \mathrm{dex}$, respectively, were employed. Additional models were calculated to confirm the interpolated values. In general the greater the number of spectrophotometric flux values, the better the resultant temperature determination.

\section{Observational data}

To assess the discrepancies of the effective temperatures and surface gravities found with the algorithm of Napiwotzki et al. (1993) and those found by comparison of fluxes and $\mathrm{H} \gamma$ profiles with ATLAS9 model predictions, 48 stars with fluxes based on the Hayes-Latham (1975) calibration of Vega mainly from the catalogs of Breger (1976) and Adelman et al. (1989) were studied. The homogeneous $u v b y \beta$ values of Hauck \& Mermilliod (1980) were employed. The $\mathrm{H} \gamma$ profiles were extracted from $20 \AA \mathrm{mm}^{-1}$ spectrograms obtained with either Reticon or CCD detectors at the Dominion Astrophysical Observatory (DAO) and had a resolution of $0.6 \AA$ (2 pixels). The exposures were flat fielded with those of an incandescent lamp placed in the Coudé mirror train as viewed through a filter to eliminate first order light. A central stop removed light from the beam in the same manner as the secondary mirror of the telescope. The spectra were rectified with the interactive computer graphics program REDUCE (Hill et al. 1982). A 3.5\% correction (Gulliver et al. 1996) was applied for scattered light along the dispersion direction.

The normalization of the $\mathrm{H} \gamma$ observations is not without problems. The continuum can be difficult to locate for late A and cooler stars and while the sensitivity of the spectrograph at the DAO changes over the $200 \AA$ interval centered on this Balmer line due to the high reflective coatings used in the Coudé mirror train. In retrospect one might be able to improve somewhat the normalization by the use of all of the observations from a single night, especially if observations were made of a range of spectral types, rather than processing each spectrum independently. This would necessitate some changes in reduction software. Alternatively if such data becomes available one might be able to use spectrophotometry having continuous wavelength coverage and a resolution of a few $\AA$ to help the normalization process.

\section{B and A star temperatures and surface gravities}

As most spectrophotometers capable of determining high quality stellar fluxes in the optical region were retired from service about twenty years ago, the candidate stars for $\mathrm{H} \gamma$ observation were those whose values were cataloged and could be observed from the DAO in Victoria. Additional selection criteria were that the spectrophotometric wavelength range must span the Balmer jump and that Strömgren indices derived from the spectrophotometry were in good accord with those values directly observed. The reddening, if any, was deduced using the computer program of Napiwotzki et al. (1993) and is uncertain to $\sim 0.005$ mag. If positive and would result in an observable change in the flux distribution, the spectrophotometric values were dereddened using those of Schild (1977). The information content of the spectrophotometric values is greater than that of the photometric values.

In this study we also wanted to avoid studying stars for which uncertainties in convection theory have observable effects on the fluxes and H $\gamma$ profiles. Smalley \& Kupka (1997) argued that the turbulent convection theory of Canuto \& Mazzitelli (1991, 1992) should be more realistic than MixingLength theory (Castelli et al. 1997). Kupka (private communication) supplied the necessary subroutine for CanutoMazzitelli convection in ATLAS9. Smalley \& Kupka (1997) indicate the discrepancies in effective temperatures and surface gravities between these theories.

As it would be useful to know where these two theories predict observable discrepancies, we found the last stellar models which exhibit minimal differences (less than 1\%) in the optical region fluxes and $\mathrm{H} \gamma$ profiles as one proceeds towards cooler temperatures. These models had the fine solar abundance opacity distribution function, a microturbulence of $2 \mathrm{~km} \mathrm{~s}^{-1}$, and 64 depths converged usually to better than $1 \%$ in flux and in flux derivative. The convergence properties of the models with Canuto \& Mazzitelli convection are often nicer than those for Mixing-Length theory. The synthetic spectra whose resolution is 500000 were broadened by the instrumental profile of the DAO long camera (Gulliver \& Hill 1990). Our comparisons were made by making plots of the $\mathrm{H} \gamma$ profiles and flux distributions that utilized most of the dimensions of letter sized pages. Initially we used a grid of models with a $500 \mathrm{~K}$ spacing in effective temperature. As the comparisons proceeded we reduced the spacing to find the onset of differences to $\pm 25 \mathrm{~K}$. Thus the coolest models with no significant differences occur at $7725 \mathrm{~K}$ for $\log g=3.00,7850 \mathrm{~K}$ for $\log g=3.25$, $8000 \mathrm{~K}$ for $\log g=3.50,8150 \mathrm{~K}$ for $\log g=3.75,8300 \mathrm{~K}$ for $\log g=4.00$, and $8475 \mathrm{~K}$ for $\log g=4.25$. These boundary values are likely to depend on microturbulence and metallicity, variables not investigated at this time. The stars studied in this paper are on the hot side of this boundary.

Table 1 contains the determinations of the temperatures and surface gravities along with the HD number and another identification, the metallicities (with $[\mathrm{m} / \mathrm{H}]=0$ being solar), the sources of the spectrophotometry, and the reddenings for 48 stars whose effective temperatures are $\geq 8000 \mathrm{~K}$. The values longward of $\mathrm{H} \alpha$ were given lower weights than other values owing to the authors' previous difficulties in obtaining simultaneous fits to them and other spectrophotometric values (see, e.g., Adelman et al. 1999). Figure 1 shows as an example the best fit for $v$ Cap. For most stars the discrepancies are less between the observations and the best theoretical fits. But in the Paschen continuum the spectrophotometric values of $\beta \mathrm{Lib}$, $\tau$ Her, and $\epsilon$ Cas produced only a fair fit to the predicted fluxes of the models. As the photometric values of $T_{\text {eff }}$ and $\log g$ for $\theta$ Vir were determined without the use of a measured $\beta$ value, they are not used for comparison with the spectrophotometrically derived values. For 22 Dra which has spectrophotometry by Schild et al. (1971), we found $T_{\text {eff }}=12500 \mathrm{~K}$ and $\log g=3.55$, but this star lacks published $u v b y \beta$ values. 
Table 1. Effective temperature and surface gravity determinations.

\begin{tabular}{|c|c|c|c|c|c|c|c|c|}
\hline$\overline{\text { Star }}$ & $\overline{\mathrm{HD}}$ & 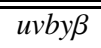 & & spectro. + & $\overline{\mathrm{H} \gamma \gamma}$ & & spectro. & reddening \\
\hline Name & number & $T_{\text {eff }}$ & $\log g$ & $T_{\text {eff }}$ & $\log g$ & {$[m / \mathrm{H}]$} & source & $E(b-y)$ \\
\hline$\tau$ Vir & 122408 & 8085 & 3.84 & 8150 & 3.75 & 0.0 & APW & 0.00 \\
\hline 95 Leo & 103578 & 8331 & 4.14 & 8300 & 3.65 & 0.0 & APW & 0.01 \\
\hline$\delta$ Her & 154494 & 8361 & 3.95 & 8500 & 4.00 & 0.0 & DP & 0.01 \\
\hline HR 6410 & 156164 & 8615 & 3.70 & 8500 & 4.00 & 0.0 & APW & 0.00 \\
\hline$\delta \mathrm{UMa}$ & 106591 & 8664 & 3.87 & 8650 & 4.10 & 0.0 & AP & 0.00 \\
\hline \multirow[t]{2}{*}{$\lambda$ Boo } & 125162 & 8925 & 4.11 & 8750 & 4.00 & 0.0 & OKE & 0.02 \\
\hline & & & & 8700 & 4.15 & -1.5 & & \\
\hline$\delta$ Aqr & 216677 & 8657 & 3.56 & 8750 & 3.50 & 0.0 & MOR & 0.00 \\
\hline \multirow[t]{2}{*}{68 Tau } & 27962 & 9025 & 3.95 & 9000 & 4.00 & 0.0 & LL & 0.00 \\
\hline & & & & 8900 & 4.00 & 0.2 & & \\
\hline \multirow[t]{2}{*}{$\lambda \mathrm{UMa}$} & 89021 & 8790 & 3.75 & 9000 & 3.75 & 0.0 & SPO & 0.00 \\
\hline & & & & 9000 & 3.75 & 0.2 & & \\
\hline$\theta$ And & 1280 & 8968 & 3.87 & 9000 & 4.00 & 0.0 & APW & 0.00 \\
\hline$\gamma \mathrm{Oph}$ & 161868 & 9388 & 4.09 & 9100 & 4.00 & 0.0 & APW & 0.02 \\
\hline$\gamma \mathrm{Gem}$ & 47105 & 9277 & 3.51 & 9150 & 3.60 & 0.0 & TAY & 0.00 \\
\hline$\epsilon \mathrm{Aqr}$ & 198001 & 9229 & 3.56 & 9200 & 3.50 & 0.0 & SPO & 0.00 \\
\hline$v$ Tau & 25490 & 9226 & 3.93 & 9250 & 4.00 & 0.0 & MOR & 0.00 \\
\hline$\theta$ Leo & 97633 & 9289 & 3.62 & 9250 & 3.60 & 0.0 & APW & 0.00 \\
\hline 27 Lyn & 67006 & 9320 & 3.77 & 9250 & 3.75 & 0.0 & A & 0.01 \\
\hline \multirow[t]{2}{*}{60 Leo } & 95608 & 9053 & 4.22 & 9250 & 4.25 & 0.0 & BVJ & 0.00 \\
\hline & & & & 9250 & 4.25 & 0.5 & & \\
\hline$\gamma \mathrm{UMa}$ & 103287 & 9361 & 3.79 & 9350 & 3.75 & 0.0 & APW & 0.00 \\
\hline$\theta$ Vir & 114330 & $9407 *$ & $3.87 *$ & 9350 & 3.50 & 0.0 & SPO & 0.00 \\
\hline 21 Lyn & 58142 & 9601 & 3.74 & 9425 & 3.75 & 0.0 & AP & 0.00 \\
\hline HR 6127 & 148330 & 9670 & 4.00 & 9500 & 3.80 & 0.0 & AP93 & 0.01 \\
\hline$\zeta$ Aql & 177724 & 9542 & 3.84 & 9500 & 3.90 & 0.0 & SPO & 0.01 \\
\hline o Peg & 214994 & 9591 & 3.64 & 9525 & 3.70 & 0.0 & A & 0.00 \\
\hline$\gamma$ Lyr & 176437 & 9674 & 2.67 & 9550 & 2.75 & 0.0 & SPO & 0.01 \\
\hline \multirow[t]{2}{*}{$\beta \mathrm{UMa}$} & 95418 & 9601 & 3.85 & 9600 & 3.80 & 0.0 & AP & 0.00 \\
\hline & & & & 9600 & 3.80 & 0.2 & & \\
\hline HR 7086 & 174262 & 9507 & 4.12 & 9600 & 4.20 & 0.0 & APW & 0.00 \\
\hline HR 5169 & 119763 & 9639 & 4.12 & 9700 & 4.15 & 0.0 & APW & 0.00 \\
\hline$\alpha \operatorname{Sex}$ & 87887 & 9862 & 3.54 & 9875 & 3.55 & 0.0 & MOR & 0.01 \\
\hline$\alpha$ Dra & 123299 & 9975 & 3.63 & 10000 & 3.50 & 0.0 & A & 0.00 \\
\hline$\sigma$ Aqr & 213320 & 10188 & 3.94 & 10100 & 3.85 & 0.0 & A & 0.00 \\
\hline $29 \mathrm{Vul}$ & 196724 & 10397 & 4.14 & 10200 & 4.00 & 0.0 & WKH & 0.00 \\
\hline$\kappa$ Сер & 192907 & 10341 & 3.64 & 10250 & 3.75 & 0.0 & APW & 0.00 \\
\hline$v$ Cap & 193432 & 10311 & 3.86 & 10250 & 4.00 & 0.0 & A & 0.00 \\
\hline $21 \mathrm{Peg}$ & 209459 & 10375 & 3.47 & 10350 & 3.55 & 0.0 & $\mathrm{AP}$ & 0.01 \\
\hline 14 Cyg & 185872 & 10953 & 4.11 & 10750 & 3.55 & 0.0 & APW & 0.00 \\
\hline 134 Tau & 38899 & 10928 & 4.01 & 10750 & 4.10 & 0.0 & A & 0.00 \\
\hline $5 \mathrm{Aqr}$ & 198667 & 11288 & 3.37 & 11125 & 3.55 & 0.0 & A & 0.01 \\
\hline$\beta \mathrm{Lib}$ & 135742 & 12036 & 3.26 & 12125 & 3.50 & 0.0 & KUB & 0.01 \\
\hline $21 \mathrm{Aql}$ & 179761 & 13029 & 3.44 & 13000 & 3.60 & 0.0 & A & 0.05 \\
\hline$\pi$ Cet & 17081 & 13174 & 3.70 & 13100 & 3.85 & 0.0 & A & 0.01 \\
\hline HR 2154 & 41692 & 14330 & 3.21 & 14500 & 3.50 & 0.0 & A & 0.02 \\
\hline$\tau$ Her & 147394 & 15022 & 3.93 & 15000 & 4.10 & 0.0 & A & 0.02 \\
\hline$\epsilon$ Cas & 11415 & 15290 & 3.85 & 15125 & 3.55 & 0.0 & SPO & 0.02 \\
\hline$\eta$ Aur & 32630 & 16887 & 4.08 & 16375 & 4.10 & 0.0 & SPO & 0.00 \\
\hline$\eta \mathrm{UMa}$ & 120315 & 17319 & 4.31 & 16900 & 4.30 & 0.0 & AP & 0.00 \\
\hline$\gamma$ Peg & 886 & 21482 & 3.92 & 21250 & 4.00 & 0.0 & A & 0.00 \\
\hline $1 \mathrm{Cas}$ & 218376 & 27334 & 4.10 & 29000 & 4.00 & 0.0 & A & 0.17 \\
\hline $10 \mathrm{Lac}$ & 214680 & 32845 & 4.61 & 33000 & 4.40 & 0.0 & KUB & 0.06 \\
\hline
\end{tabular}

Notes: Breger (1976) converted data from DP, KUB, MOR, OKE, SPO, and WKH to the Hayes \& Latham (1975) calibration of Vega. The spectrophotometric sources are: A = Adelman (1978), AP = Adelman \& Pyper (1983), AP93 = Adelman \& Pyper (1993), APW = Adelman et al. (1980), BVJ = Böhm-Vitense \& Johnson (1978), DP = Dickens \& Penny (1971), KUB = Kubiak (1973), LL = Lane \& Lester (1980), MOR $=$ Gutierrez-Moreno et al. (1968), OKE = Oke (1964), SPO = Schild et al. (1971), TAY = Taylor (1984), and WKH = Wolff et al. $(1968)$. $*$ = estimated with predicted $\beta$ value. 


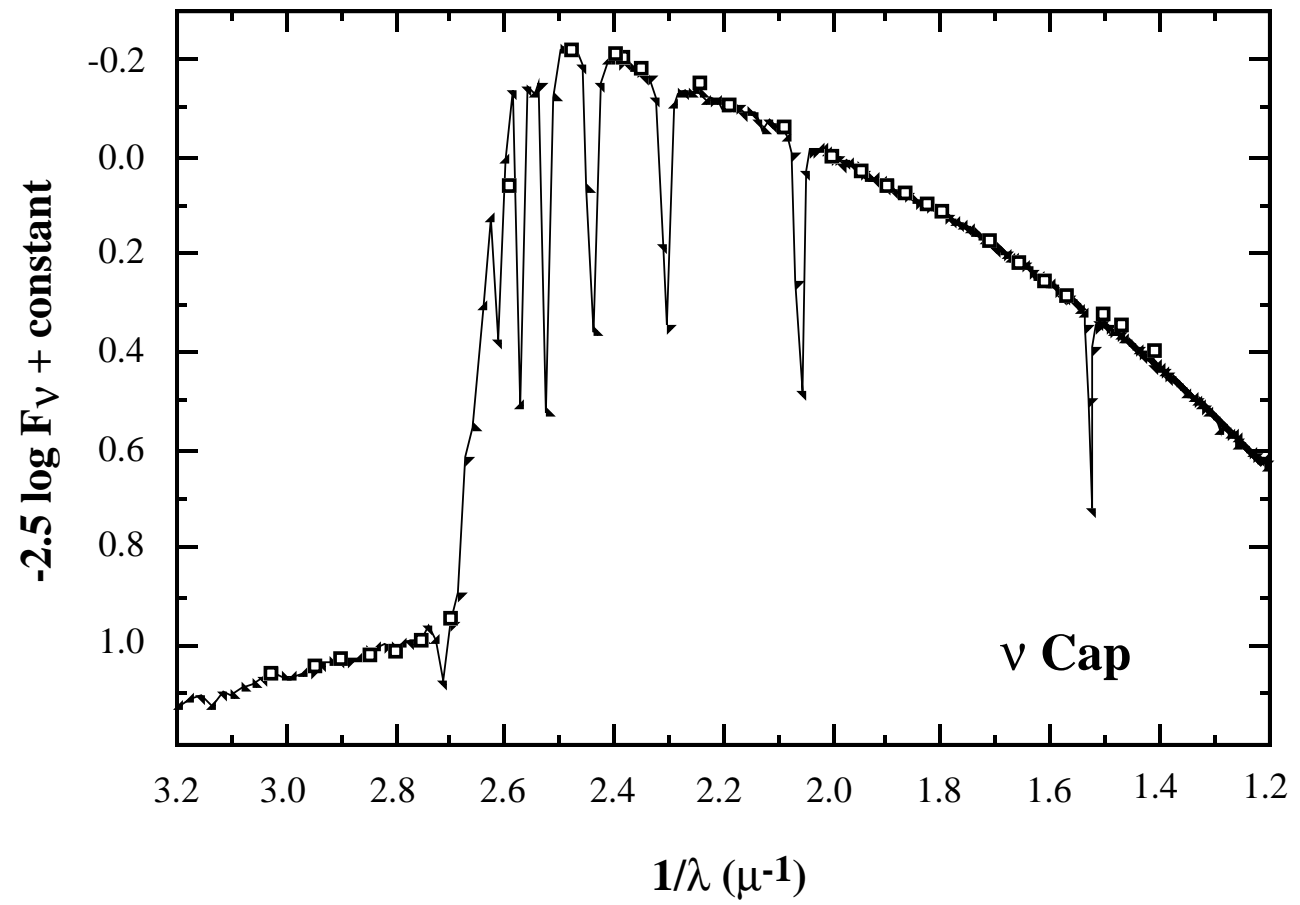

Fig. 1. The spectrophotometric fluxes for $v$ Cap (open squares) compared with the predictions of a solar composition $T_{\text {eff }}=10250 \mathrm{~K}, \log g=$ 4.00 ATLAS9 model atmosphere with a microturbulence of $0 \mathrm{~km} \mathrm{~s}^{-1}$ (thin line connecting solid triangles). The axes are inverse microns and the magnitude of the flux in frequency units in ergs $\mathrm{cm}^{-2} \mathrm{~s}^{-1} \mathrm{~Hz}^{-1}$. The constant was chosen to make the flux at $5000 \AA$ be 0.0 for the observations. The theoretical fluxes were shifted for the best match over as much of the observed wavelength range as possible.

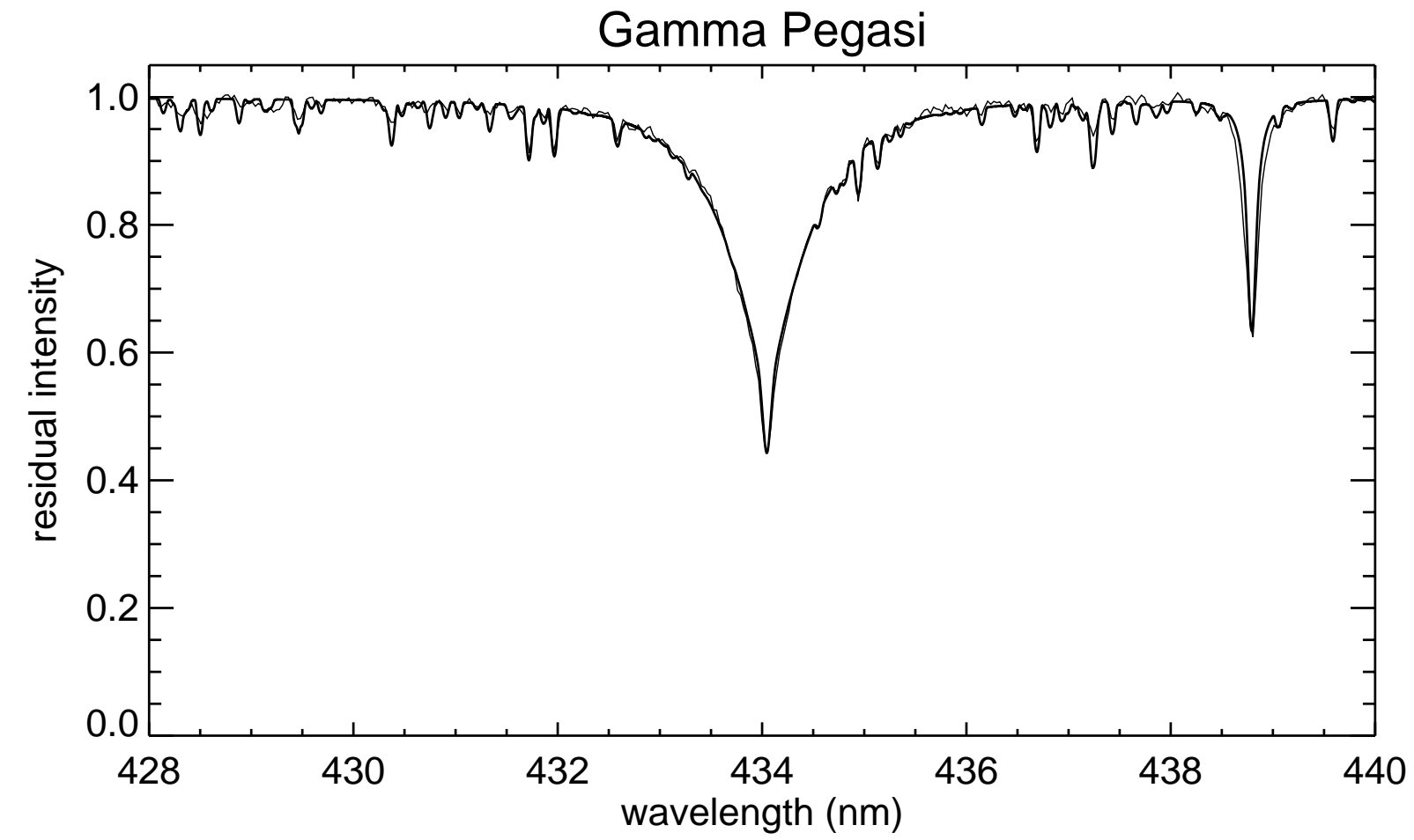

Fig. 2. The synthesized spectrum of $\gamma$ Peg (thicker line) centered at $\mathrm{H} \gamma$ calculated with a solar composition $T_{\text {eff }}=21250 \mathrm{~K}$, log $g=4.00$ ATLAS9 model atmosphere with a microturbulence of $0 \mathrm{~km} \mathrm{~s}^{-1}$ (thinner line).

For most stars the synthesized $\mathrm{H} \gamma$ regions match the observations quite well, especially the Balmer line, but not perfectly. Three representative examples are shown in Figs. 2-4 with the thicker lines being the synthesized spectra and the thinner lines the observations. For $\gamma$ Peg, the He I line $\lambda 4388$ is not satisfactorially synthesized and for $\gamma \mathrm{Gem}$ the metal lines in the synthesized spectrum are slightly too deep. For $10 \mathrm{Lac}$, the worst case in fitting, the Balmer line profile fit was to the $\mathrm{H} \gamma$ line wings 


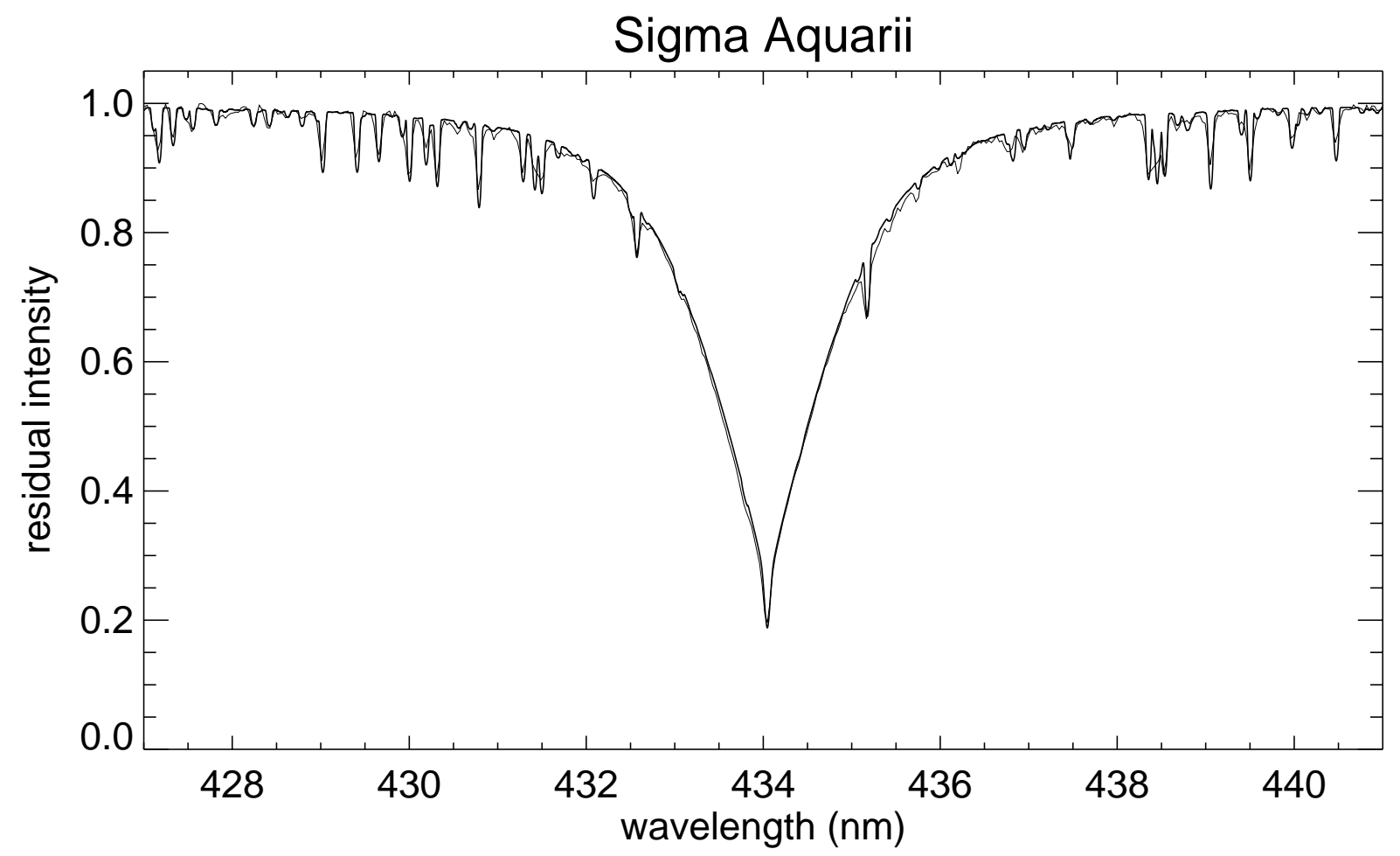

Fig. 3. The synthesized spectrum of $\sigma$ Aqr (thicker line) centered at $\mathrm{H} \gamma$ calculated with a solar composition $T_{\text {eff }}=10100 \mathrm{~K}, \log g=3.85$ ATLAS9 model atmosphere with a microturbulence of $1 \mathrm{~km} \mathrm{~s}^{-1}$ (thinner line).

with the observed core being deeper, especially in the central $5 \AA$ A by about $5 \%$, which may represent non-LTE effects while for 60 Leo models with a $4 \mathrm{~km} \mathrm{~s}^{-1}$ microturbulence were used consistent with the analysis by Adelman et al. (1999).

For 48 stars the average difference in $T_{\text {eff }}$ (photometric minus spectrophotometric- $\mathrm{H} \gamma$ value) is $29 \pm 293 \mathrm{~K}$. For those stars with $8000 \mathrm{~K} \leq T_{\text {eff }} \leq 10050 \mathrm{~K}$, we find a difference of $25 \pm 118 \mathrm{~K}$ and for stars with $10050 \mathrm{~K} \leq T_{\text {eff }} \leq 17000 \mathrm{~K}$, $76 \pm 105 \mathrm{~K}$. This suggests a slight decrease of the derived temperature scale which is not unexpected as it reflects an increase in the metal line opacity for ATLAS9 relative to ATLAS6 models. Figure 5 shows the difference $\left(\Delta T_{\text {eff }}(\mathrm{K})\right)$ between the effective temperatures as derived using photometry and spectrophotometry as a function of the photometric temperature $\left(T_{\text {eff }}(u v b y \beta)(\mathrm{K})\right)$.

The mean difference in $\log g$ (photometric minus spectrophotometric- $\mathrm{H} \gamma$ value) for 47 stars is $-0.007 \pm$ 0.169 dex. The scatter is similar to the uncertainty found by Lemke (1989) 0.20 dex. But two stars, 95 Leo and 14 Cyg, have $\log g$ values with rather large discrepancies, 0.49 dex and $0.56 \mathrm{dex}$, respectively. Together they have a 0.02 dex effect on the average. Figure 6 shows the difference $(\Delta \log g(\operatorname{dex}))$ between the surface gravity as derived using photometry and from spectrophotometry and the $\mathrm{H} \gamma$ profile as function of the photometric temperature $T_{\text {eff }}(u v b y \beta)(\mathrm{K})$. It is best described as a scatter diagram. Since the discrepancies in the temperatures are small relative to the derived temperatures, the basic agreement of the surface gravity scales is not unexpected.

In Table 2 we compare the calibration or "known" temperatures that Napiwotzki et al. (1993) used from
Table 2. Comparison of effective temperature and surface gravity determinations.

\begin{tabular}{lrrr}
\hline \hline & & $T_{\text {eff }}$ & \\
Star & "known" & $u v b y \beta$ & spectro. $+\mathrm{H} \gamma$ \\
\hline$\gamma$ Gem & 9240 & 9277 & 9150 \\
$\beta$ UMa & 9170 & 9601 & 9600 \\
$\nu$ Cap & 9950 & 10311 & 10250 \\
134 Tau & 10790 & 10928 & 10750 \\
$\pi$ Cet & 12820 & 13174 & 13100 \\
$\eta$ Aur & 17580 & 16887 & 16375 \\
\hline
\end{tabular}

Code et al. (1976), Beeckmans (1977), and Malagini et al. (1986) with those from their $u v b y \beta$ calibation and our values. Smalley \& Dworestsky (1995) re-evaluated the fundamental values of Code et al. (1976) using more recent flux measurements and found no significant changes. In general our results agreed better with the photometric values than the calibrator values and exhibit agreement with the calibrator similar to that of the photometric values. However this comparison can be done only for a few calibrators. How the nonunformity of the spectrophotometric data affects these results is unclear and is a possible source of some differences as the data quality is not uniform among the spectrophotometric sources.

\section{Conclusions}

To substantially decrease the errors from this type of study we need a set of uniform high quality optical flux measurements with near continuous coverage for many more stars. Small systematic differences in effective temperature, but not in surface 
Gamma Geminorum

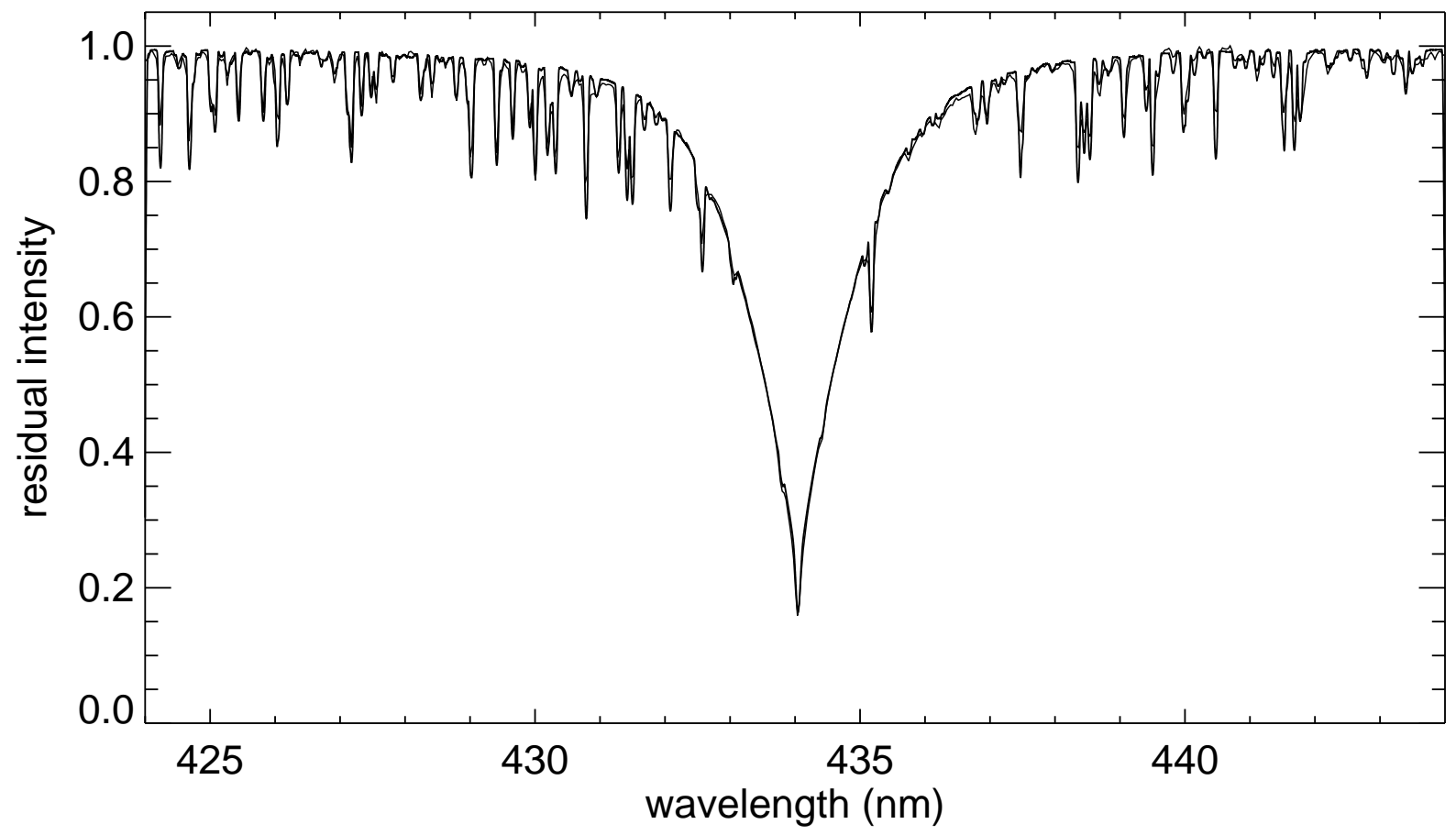

Fig. 4. The synthesized spectrum of $\gamma$ Gem (thicker line) centered at $\mathrm{H} \gamma$ calculated with a solar composition $T_{\text {eff }}=9150 \mathrm{~K}$, log $g=3.60$ ATLAS9 model atmosphere with a microturbulence of $2 \mathrm{~km} \mathrm{~s}^{-1}$ (thinner line).

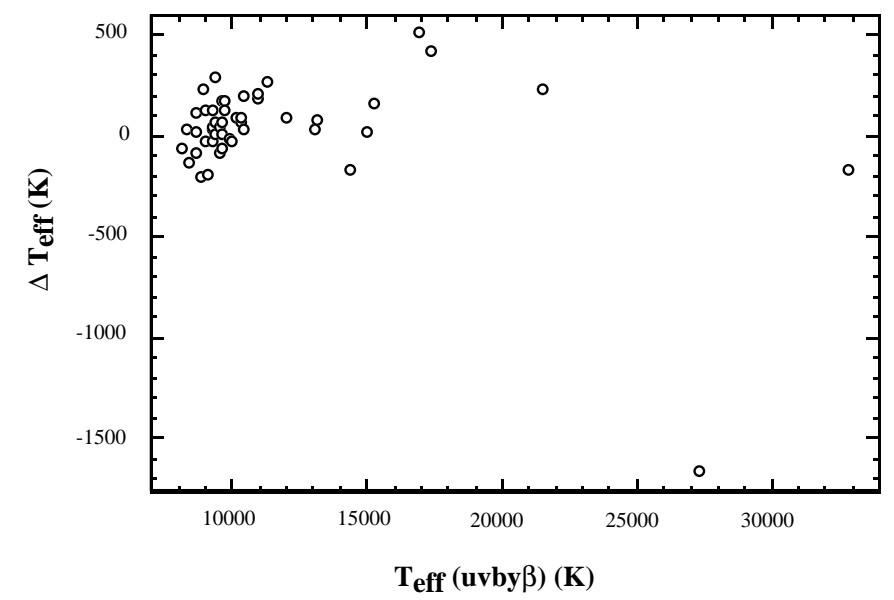

Fig. 5. The differences $\left(\Delta T_{\text {eff }} \quad(\mathrm{K})\right)$ (photometric minus spectrophotometric- $\mathrm{H} \gamma$ values) of the effective temperatures as a function of the photometric temperature $T_{\text {eff }}(u v b y \beta)(\mathrm{K})$.

gravity have been found between matching optical spectrophotometery and $\mathrm{H} \gamma$ profiles with the predictions of ATLAS9 solar composition model atmospheres and those derived from Strömgren $u v b y \beta$ photometry based on ATLAS6 model atmospheres. These discrepancies have been attributed to small differences in the line opacities. In performing elemental abundance analyses, their effects will be small. But as one must do many steps as well as possible, the total effect of many such differences might be substantial.

Acknowledgements. SJA's contribution to this paper was support in part by grants from The Citadel Foundation as was that of KER.

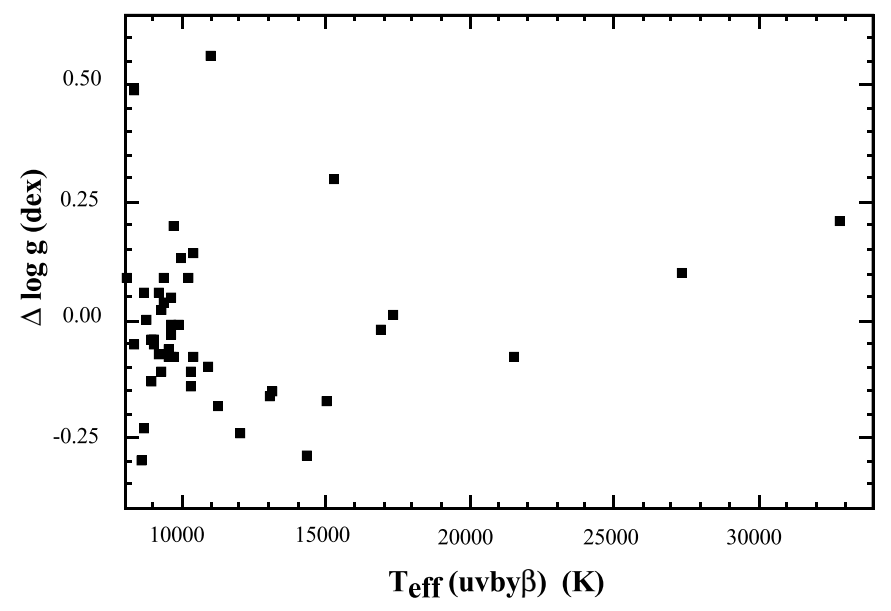

Fig. 6. The differences $(\Delta \log g$ (dex)) (photometric minus spectrophotometric- $\mathrm{H} \gamma$ values) of the surface gravities as a function of the photometric temperature $T_{\text {eff }}(u v b y \beta)(\mathrm{K})$.

Some $\mathrm{H} \gamma$ observations at the DAO were made by Frank Younger and Charles Perry. We thank F. Kupka for his implementation of CanutoMazzitelli convection in ATLAS9.

\section{References}

Adelman, S. J. 1978, ApJ, 222, 547

Adelman, S. J. 1999, MNRAS, 310, 146

Adelman, S. J., \& Pyper, D. M. 1983, ApJ, 266, 732

Adelman, S. J., \& Pyper, D. M. 1993, A\&AS, 101, 393 
Adelman, S. J., Pyper, D. M., \& White, R. E. 1980, ApJS, 43, 491

Adelman, S. J., Pyper, D. M., Shore, S. N., White, R. E., \& Warren, W. H. Jr 1989, A\&AS, 81, 221

Adelman, S. J., Caliskan, H., Cay, T., Kocer, D., \& Gokmen Tektunali, H. 1999, MNRAS, 305, 591

Beeckmans, F. 1977, A\&A, 60, 1

Böhm-Vitense, E., \& Johnson, P. 1978, ApJ, 225, 514

Breger, M. 1976, ApJS, 32, 7

Canuto, V. M., \& Mazzitelli, I. 1991, ApJ, 370, 295

Canuto, V. M., \& Mazzitelli, I. 1992, ApJ, 389, 724

Castelli, F., Gratton, R. G., \& Kurucz, R. L. 1997, A\&A, 318, 841

Code, A. D., Davis, J., Bless, R. C., \& Hanbury Brown, R. 1976, ApJ, 203, 417

Dickens, R. J., \& Penny, A. J. 1971, MNRAS, 153, 287

Gulliver, A. F., \& Hill, G. 1990, PASP, 102, 1200

Gulliver, A. F., Hill, G., \& Adelman, S. J. 1996, in 5th Vienna Symp. on Stellar Atmospheres and Spectrum Synthesis, ed. S. J. Adelman, F. Kupka, \& W. W. Weiss (San Francisco, Astron. Soc. Pacific), ASP Conf. Ser., 108, 232

Gutierrez-Moreno, A., Moreno, H., \& Stock, J. 1968, Univ. Chile Dept. Astron. Pub., 8, 127

Hauck, B., \& Mermilliod, M. 1980, A\&AS, 40, 1

Hayes, D. S., \& Latham, D. W. 1975, ApJ, 197, 593
Hill, G., Fisher, W. A., \& Poeckert, R. 1982, Publ. Dom. Astrophys. Obs. Victoria, 16, 27

Lane, M. C., \& Lester, J. B. 1980, ApJ, 228, 210

Lemke, M. 1989, A\&A, 225, 125

Kubiak, M. 1973, Acta Astr., 23, 23

Kurucz, R. L. 1979, ApJS, 40, 1

Kurucz, R. L. 1993, Atlas9 Stelllar Atmosphere Programs and 2 km/s grid, Kurucz CD-Rom No. 13 (Smithsonian Astrophysical Observatory, Cambridge)

Kurucz, R. L., \& Avrett, E. H. 1981, Smithsonian Astrophysical Observatory Report, 391

Malagnini, M. L., Morossi, C., Rossi, L., \& Kurucz, R. L. 1986, A\&A, 162,140

Moon, T. T., \& Dworetsky, M. M. 1985, MNRAS, 217, 305

Napiwotzki, R., Schönberner, D., \& Wenske, V. 1993, A\&A, 268, 653

Oke, J. B. 1967, ApJ, 150, 513

Ribas, I., Jordi, C., Torra, J., \& Gimenez, A. 1997, A\&A, 327, 207

Schild, R. E. 1977, AJ, 82, 337

Schild, R., Peterson, D. M., \& Oke, J. B. 1971, ApJ, 166, 95

Smalley, B., \& Dworetsky, M. M. 1995, A\&A, 293, 446

Smalley, B., \& Kupka, F. 1997, A\&A, 328, 349

Taylor, B. J. 1984, ApJS, 54, 256

Wolff, S. C., Kuhi, L. V., \& Hayes, D. S. 1968, ApJ, 152, 871 